\title{
Comparison of experiment and models of geodesic acoustic mode frequency and amplitude geometric scaling in ASDEX Upgrade
}

\author{
P.Simon $^{1,2,3}$, G.D.Conway ${ }^{1}$, U.Stroth ${ }^{1,4}$, A.Biancalani ${ }^{1}$, \\ F.Palermo ${ }^{1}$, and the ASDEX Upgrade Team ${ }^{1}$ \\ ${ }^{1}$ Max-Planck-Institut für Plasmaphysik, 85748 Garching, Germany \\ ${ }^{2}$ Institut für Grenzflächenverfahrenstechnik und Plasmatechnologie, Universität \\ Stuttgart, 70569 Stuttgart, Germany \\ ${ }^{3}$ Institut Jean Lamour, Université de Lorraine, 54011 Nancy, France \\ ${ }^{4}$ Physik-Department E28, Technische Universität München, 85747 Garching, \\ Germany
}

\begin{abstract}
In a set of dedicated ASDEX Upgrade shape-scan experiments, the influence of plasma geometry on the frequency and amplitude behaviour of the geodesic acoustic mode (GAM), measured by Doppler reflectometry, is studied. In both limiter and divertor configurations, the plasma elongation was varied between circular and highly elongated states $(1.1<\kappa<1.8)$. Also, the edge safety factor was scanned between $3<q<5$. The GAM frequency $\omega_{\mathrm{GAM}}$ and amplitude are used to test several models (heuristic, fluid and gyrokinetic based), which incorporate various plasma geometry effects. The experimentally observed effect of decreasing $\omega_{\mathrm{GAM}}$ with increasing $\kappa$ is predicted by most models. Other geometric factors, such as inverse aspect ratio $\epsilon$ and Shafranov shift gradient $\Delta^{\prime}$ are also seen to be influential in determining a reliable lower $\omega_{\mathrm{GAM}}$ boundary. The GAM amplitude is found to vary with boundary elongation $\kappa_{b}$ and safety factor $q$. The collisional damping is compared to multiple models for the collisionless damping. Collisional damping appears to play a stronger role in the divertor configuration, while collisional and collisionless damping both may contribute to the GAM amplitude in the limiter configuration.
\end{abstract}

PACS numbers: 52.25.Fi, 52.55.Fa, 52.35.Ra,

Submitted to: Plasma Phys. Control. Fusion 


\section{Introduction}

One of the more important research topics in the field of magnetic confinement fusion is the study of anomalous (or turbulent) transport, which is believed to be a major contributor to particle and energy losses and thus plays a fundamental role in the global confinement of a plasma [1, 2]. Any mechanism involved in determining the saturated level of turbulent transport is therefore of great interest. One such mechanism in toroidal plasmas (as well as other turbulent systems such as rotating planets or the sun) is the zonal flow $(\mathrm{ZF})$.

Zonal flows are radially localised axisymmetric $E \times B$ shear flows ( $m=n=0$, with poloidal and toroidal mode numbers $m$ and $n$ ) [3]. These quasi-stationary modes $\left(\omega_{\mathrm{ZF}} \approx 0\right)$ are driven by non-linear interactions within the turbulence and thus can self-regulate the plasma transport via flow shearing [4]. When coupled to an $m= \pm 1$ pressure side-band mode, the ZF gives rise to a resonant oscillation called the geodesic acoustic mode (GAM) which can also contribute to flow-shear stabilisation of turbulence.

The GAM's eigenfrequency in a simplified circular plasma geometry was first presented by Winsor et al.:

$$
\omega_{\mathrm{GAM}}=\sqrt{2+q^{-2}} c_{s} / R_{0},
$$

where $c_{s}=\sqrt{\left(T_{e}+\gamma_{i} T_{i}\right) / m_{i}}$ is the sound velocity and $R_{0}$ is the major plasma radius. $T_{e}$ and $T_{i}$ are the electron and ion temperatures respectively, $q$ is the safety factor, $m_{i}$ is the ion mass of the plasma and $\gamma_{i}$ the ion specific heat ratio (typically understood to be of the order of 1) [5]. For $q \gg 1$ in the tokamak plasma edge this reduces to $\omega_{\mathrm{GAM}}=\sqrt{2} c_{s} / R_{0}$.

In addition to the GAM frequency it is also important to investigate the behaviour of the GAM amplitude $A_{\mathrm{GAM}}$, as it determines the effect that the GAM may have on the $E \times B$ shearing rate. Only if the amplitude is sufficiently large to cause displacements comparable to the turbulent structure size may the GAM play a significant role in the moderation of turbulence. It has been found that the amplitude is also dependent on plasma parameters such as the safety factor $[6,7]$.

Experimentally, GAMs have been observed in many tokamak plasmas $[6,8,9$, $10,11,12,13]$, often in reasonably good agreement with Winsor's predicted frequency, especially in circular plasma discharges. However, for experiments with non-circular plasmas in a divertor $\mathrm{X}$-point configuration, deviations from the Winsor scaling were found $[6,11,14,15]$. In particular, larger plasma boundary elongations $\kappa_{b}$ were found to decrease the GAM frequency, which was expressed in a heuristic scaling law of the form $\omega_{\mathrm{GAM}} \propto 1 /\left(1+\kappa_{b}\right)$. Extending on the previous theoretical predictions and these experimental results, a number of more involved models for the GAM frequency scaling have been proposed in recent years, typically including plasma geometry factors such as the safety factor $q$, the shear of the elongation $s_{\kappa}$ or the Shafranov shift gradient $\Delta^{\prime}[16,17,18,19]$. While Conway's heuristic scaling [14] was determined from a database of ASDEX Upgrade discharges containing experiments both at large and at 
small elongation, this article is based on a new set of specially designed experiments, where the shape was varied from $\kappa_{b}=1.1$ to 1.8 in both divertor and limiter discharges. The new experimental results for the GAM frequency are used to test the range of validity of Winsor's simplified fluid scaling, the heuristic model as well as electrostatic fluid and gyrokinetic models derived by Angelino [19] and Gao [17]. Electromagnetic effects are also discussed. The test of these analytical models, which incorporate the effects of $\kappa_{b}$ and $q$, against an extensive single-machine database is the focus of section 4 . The amplitude scaling with respect to $\kappa$ and $q$, as well as the role of collisional and collisionless damping in the region of GAM activity are discussed in section 5. The paper starts with descriptions of the experiments and the data analysis in sections 2 and 3 , respectively.

\section{Diagnostic and experimental setup}

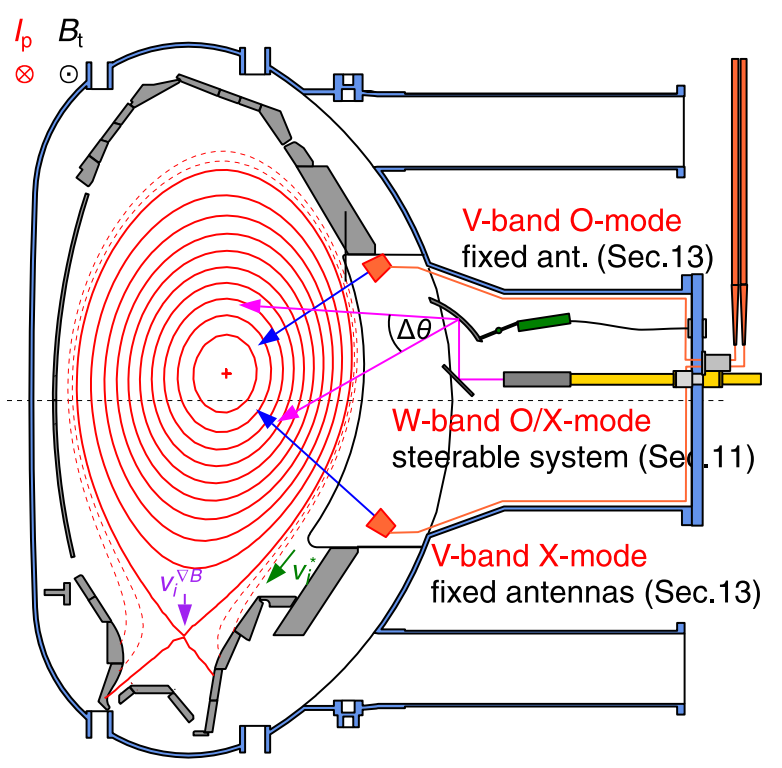

Figure 1. Schematic cross-section of ASDEX Upgrade during a divertor discharge, highlighting the measurement locations of the Doppler reflectometer systems

Since geodesic acoustic modes are a type of flow perturbation, they are well suited to be studied using microwave Doppler reflectometry. Doppler reflectometry works by launching a microwave beam into the plasma at a specific tilt angle $\theta_{0}$ with respect to the flux surface. The beam propagates into the plasma until it meets a cutoff. Due to Bragg scattering by turbulent structures in the cutoff region, part of the signal may be backscattered towards the launch antenna. For backscattering to occur, the local perpendicular wavenumber $k_{\perp}$ of the turbulence must fulfill the condition $k_{\perp}=-2 k_{0} N_{\perp}\left(\approx 2 k_{0} \sin \theta_{0}\right.$ for flat cutoffs), where $k_{0}$ is the launched beam wavenumber and $N_{\perp}$ is the plasma refractive index at the reflection layer. Due to the movement of the turbulent structures (i.e. the plasma flow), the received signal will 
be Doppler-shifted with a frequency $2 \pi f_{D}=\mathbf{v} \cdot \mathbf{k} \approx v_{\perp} k_{\perp}$ (where $k_{\|} \ll k_{\perp}$ ). Here, $v_{\perp}$ is the perpendicular velocity of the turbulent structure which is composed of the $E \times B$ background flow of the plasma and the turbulent phase velocity (considered to be negligible): $v_{\perp}=v_{E \times B}+v_{p h} \approx v_{E \times B}$. [20,21] Thus, oscillating $E \times B$ flows such as GAMs will appear as fluctuations in the Doppler shift $f_{D}$.

Three Doppler reflectometry systems are currently in operation on the ASDEX Upgrade tokamak (AUG), as shown in figure 1: two fixed-tilt antenna V-band systems (50-75 GHz, with O- and X-mode polarization) [22] and one W-band system (75$108 \mathrm{GHz}$ ) with an adjustable tilt angle on the tokamak low-field-side [23]. The $20 \mathrm{MHz}$ sampling rate of the heterodyne receiver in-phase $(I=A \cos \phi)$ and quadrature $(Q=A \sin \phi)$ detector signals, using a 12 bit ADC, allows the investigation of velocity fluctuations and radial electric field perturbations with high temporal resolution. The perpendicular wavenumber $k_{\perp}$ as well as the measurement location are obtained with the beam tracing code TORBEAM [24] for each probing frequency, using a fitted density profile and high resolution equilibrium reconstruction [21]. The two V-band reflectometers can also be coupled together on the same bistatic antenna pair, in order to launch and receive from the same poloidal angle and to allow radial correlation Doppler reflectometry measurements [25].

ASDEX Upgrade has a major radius $R_{0}=1.65 \mathrm{~m}$ and a minor radius $a=0.5 \mathrm{~m}$. The tokamak is typically operated within lower X-point divertor configuration, however, the flexible shape control also allows for limiter experiments.

In order to study the influence of the plasma elongation $\kappa$ on the GAM, a set of special shape-scan experiments were performed. During a single deuterium plasma discharge, the boundary elongation $\kappa_{b}$ was substantially varied. Figure 2 (bottom) shows a case in which a lower single divertor configuration was modified from $\kappa_{b}=1.77$ to $\kappa_{b}=1.44$. The counterpart is shown in figure 2 (top), where a nearly circular limiter plasma $\left(\kappa_{b}=1.12\right)$ is stretched to a higher elongation $\left(\kappa_{b}=1.69\right)$. This set of experiments closes the gap in previous AUG experiments that featured mainly low- $\kappa$ limiter discharges and high- $\kappa$ divertor discharges, allowing to separate the influence of both the plasma elongation as well as the configuration, e.g. the presence of an X-point null field. Overlayed in figure 2 are computed ray-traces for the lowest and highest probe frequencies in $\mathrm{X}$-mode that are used in these experiments. The corresponding launch/receiver antennas are below the midplane, as pictured in figure 1.

Figure 3 shows time traces of typical plasma quantities from one of the limiter discharges. Using control coils, the plasma boundary elongation $\kappa_{b}$ is steadily increased throughout the discharge, from 1.1 to approximately 1.7. At the same time, the plasma current $I_{p}$ is increased from 0.6 MA to $1.0 \mathrm{MA}$ in order to maintain the edge safety factor at a constant $q_{95} \approx 4$. It was also attempted to keep the line-averaged density at a constant level of $n_{e} \approx 2.5 \times 10^{19} \mathrm{~m}^{-3}$ in order to maintain the Doppler measurements in the same radial region, but small variation between 2 and $3 \times 10^{19} \mathrm{~m}^{-3}$ could not be avoided. The edge density near the separatrix or last closed flux surface was typically close to $n_{\text {edge }} \approx 0.5 \times 10^{19} \mathrm{~m}^{-3}$. In this discharge the magnetic field strength was kept 

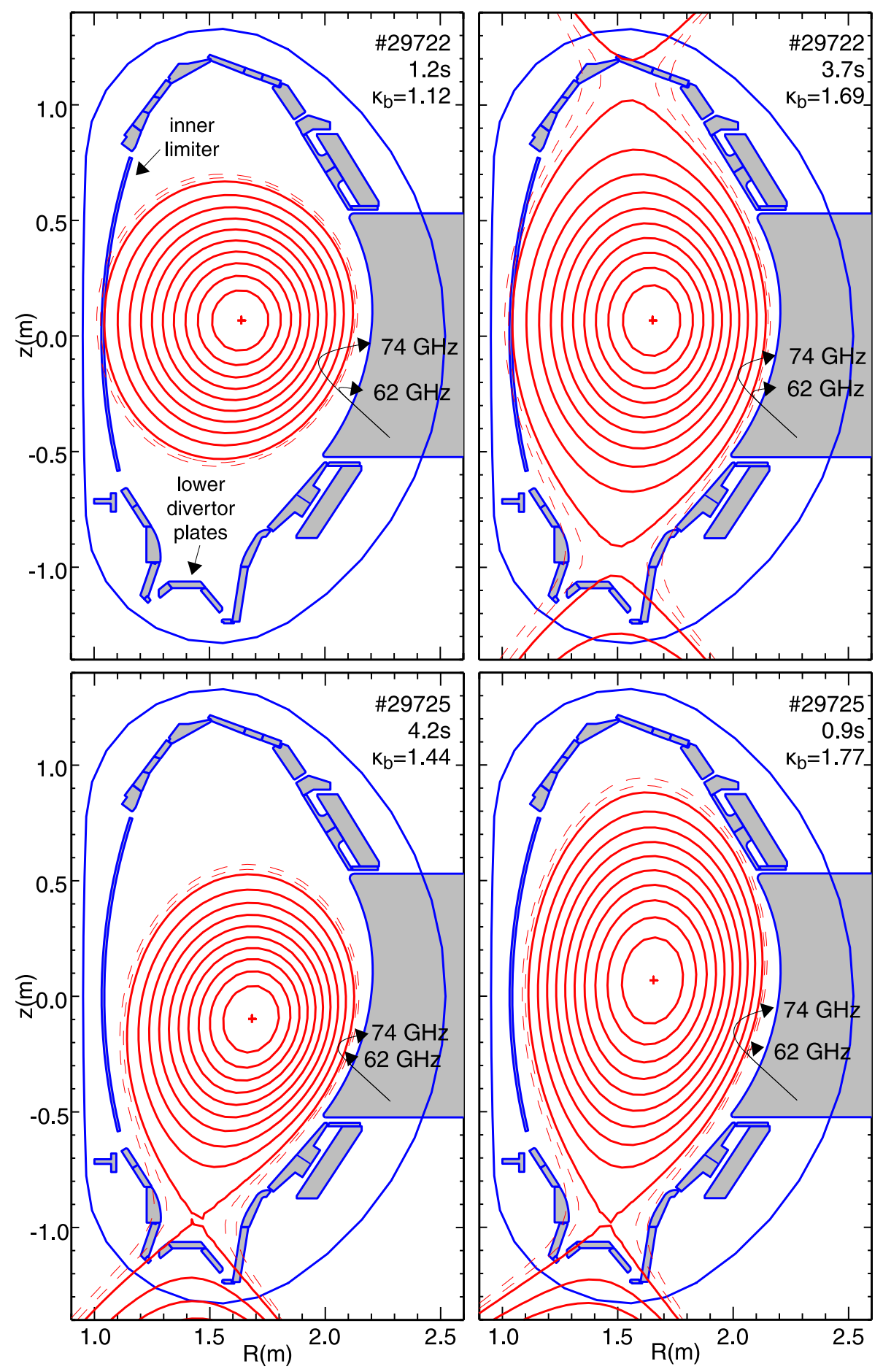

Figure 2. Plasma shape during beginning and ending of two shape-scan discharges. Top: Limiter discharge \#29722. Bottom: Divertor discharge \#29725. The red surfaces indicate constant poloidal flux $\Psi_{\text {pol }}$. The black lines indicate the probing range and measurement locations of the Doppler reflectometer.

constant at $B=-2.4 \mathrm{~T}$ and a moderate electron cyclotron resonance heating power of 0.4 MW was applied. These values of magnetic field and density were chosen to allow for X-mode Doppler measurements in the edge region of the plasma, where GAMs are 


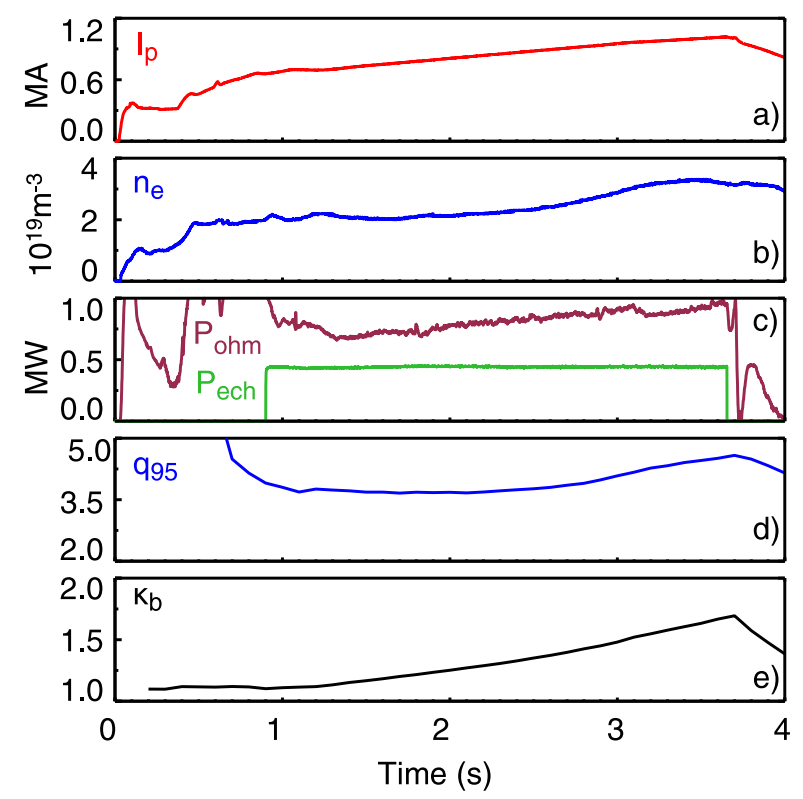

Figure 3. Time traces of AUG shape-scan discharge \#29722, showing the evolution of a) plasma current $I_{p}$, b) central line averaged density H1, c) Ohmic and ECRH heating power, d) edge safety factor $q_{95}$ and e) boundary elongation $\kappa_{b}$. $B_{T}$ was kept constant at $-2.4 \mathrm{~T}$.

typically observed during L-mode discharges at ASDEX Upgrade. Specifically, GAMs are usually detected inwards from the region of the radial electric field minimum at the plasma edge toward the $n_{e}$ pedestal top, and never outside of the separatrix or last closed flux surface. The location and range of GAM measurements, i.e. slightly below the midplane on the low field side edge, can also be seen in figure 2. The poloidal angle of beam incidence $\theta$, as well as the poloidal plasma location, remain roughly constant in all configurations.

Figure 4 shows typical edge plasma radial profiles of electron temperature $T_{e}$, density $n_{e}$ and safety factor $q$ during low- and high-elongation phases of a limiter discharge. The radial range in which GAMs are detected is slightly wider in the case of low boundary elongation.

To study the radial structure of the GAMs, the Doppler probing frequency is stepped, with one radial sweep consisting of 10-13 steps separated by $1 \mathrm{GHz}$, each lasting $15 \mathrm{~ms}$. The measurement locations were reconstructed using fitted density profiles from the profile reflectometer and Lithium-beam diagnostics. The electron temperature $T_{e}$, which is important for comparison with the theoretical models, was measured with the Thomson scattering and electron cyclotron emission (ECE) diagnostics. Experimental measurements of the ion temperature were not available in all cases and have been scaled from similar discharges. From previous experiments, for measurements towards the plasma pedestal top, $T_{i} \approx T_{e}$ was assumed, whereas in divertor discharges closer towards the separatrix, the ion temperature can rise to $T_{i} \approx 1.2 T_{e}$. [26]

For the comparison of GAM frequency and amplitude measurements to theoretical 

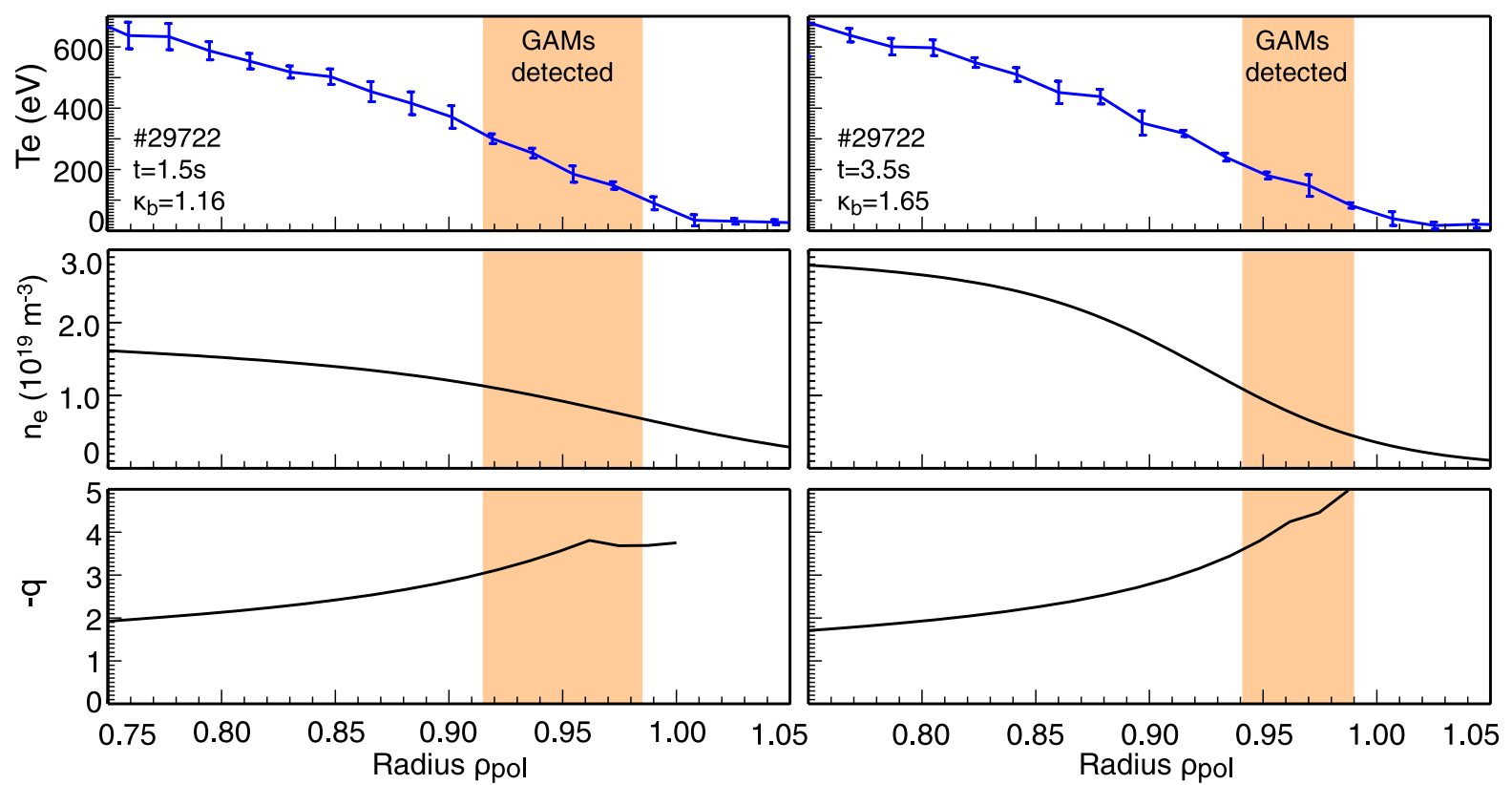

Figure 4. Radial profiles of electron temperature $T_{e}$ (top), density $n_{e}$ (middle) and safety factor q (bottom) in limiter discharge \#29722. Left: $t=1.5 \mathrm{~s}, \kappa_{b}=1.16$. Right: $t=3.5 \mathrm{~s}, \kappa_{b}=1.65$. The radial region in which GAMs were detected is highlighted.

models a database consisting of 59 radial sweeps, taken during three shape-scan discharges, was evaluated. During each radial sweep the strongest detected GAM peak (or none, if no clear GAM peak could be determined) is used in the analysis. The range of plasma boundary elongation in the database is $1.13<\kappa_{b}<1.78$, GAMs are detected in the radial range of $0.92<\rho_{\text {pol }}<0.99$ at densities of $0.6 \times 10^{19} \mathrm{~m}^{-3}<n_{e}<1.3 \times 10^{19} \mathrm{~m}^{-3}$, the local safety factor $q$ varies between 3 and 5 . Investigations of the radial GAM structure and possible GAM propagation were made during the same experiments, but are beyond the scope of this article and will be presented in a future publication.

\section{Data analysis}

The extraction of the GAM properties from the raw Doppler reflectometer signal is a multi-step process, as shown in figure 5. First, the Doppler shift frequency $f_{D}$ and amplitude $A_{D}$ are calculated from the IQ signal. The perpendicular velocity $v_{\perp}$ is then calculated from $f_{D}$ and the relative turbulence amplitude $\delta n^{2}$ at the probed $k_{\perp}$ is proportional to $A_{D}$. In order to measure GAMs, it is necessary to have a high-resolution time series $f_{D}(t)$. Therefore, $f_{D}$ and $A_{D}$ are calculated from a small window of the complete IQ signal, which slides forward in time. Finally, an average power spectrum is calculated from $f_{D}(t)$. GAMs appear as distinct peaks in this frequency spectrum, usually in the range of $5-25 \mathrm{kHz}$ for AUG L-mode discharge conditions.

The conventional way to determine the Doppler shift from the raw IQ signal is by calculating the power spectrum $S_{f}(f)$ with a Fast Fourier Transform (FFT) algorithm 

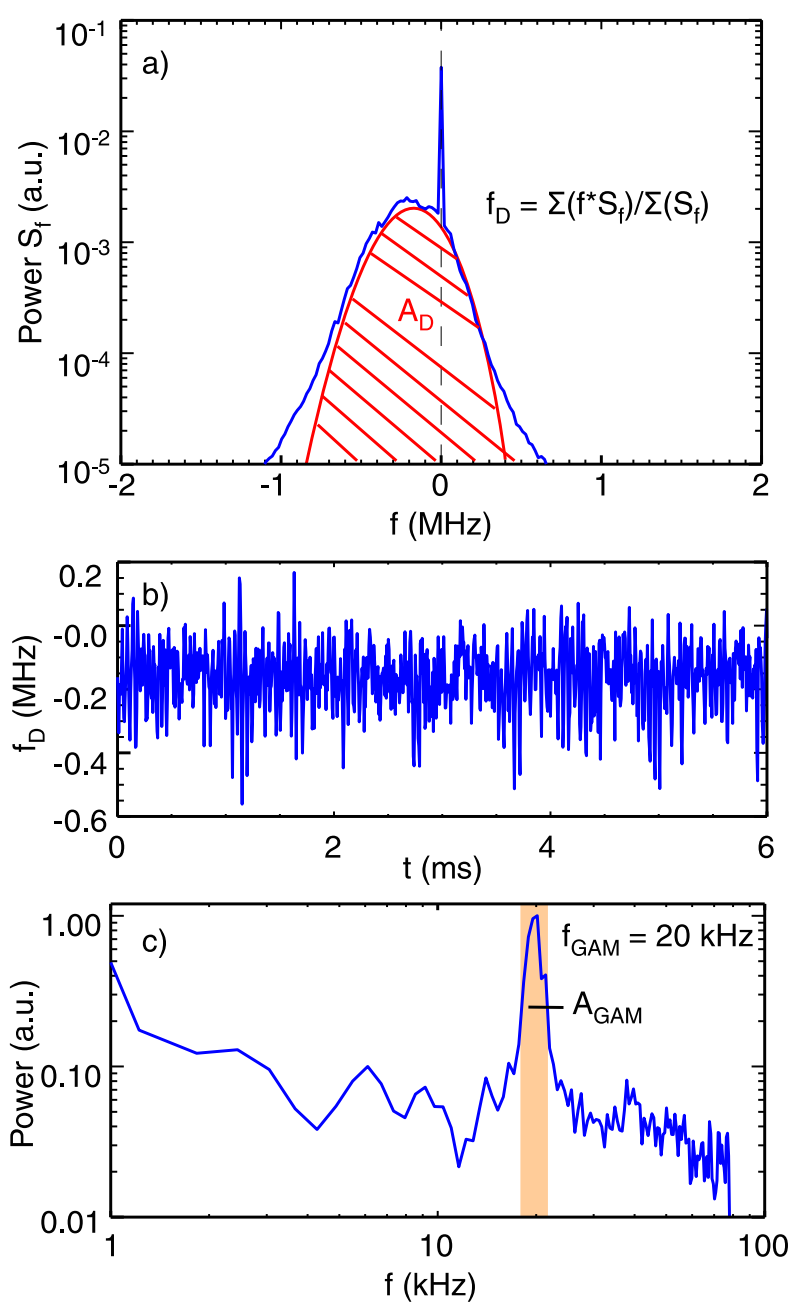

Figure 5. Illustration of the GAM detection from reflectometer data. a) Power spectrum calculated from a window of the raw data, exhibiting a clear Doppler shift. b) Time series of the determined Doppler shift frequency (proportional to the flow velocity). c) Power spectrum of the $f_{D}$ time series, showing a strong GAM peak at 20 $\mathrm{kHz}$.

and computing a weighted mean: $f_{D}=\sum f \cdot S_{f}(f) / \sum S_{f}(f)$. Typically the original data window (10-15 ms at $20 \mathrm{MHz}$ sampling rate) is split into sub-windows of 256 points (or $12.8 \mu \mathrm{s})$, with $50 \%$ overlap, for this calculation. The resulting time series $f_{D}(t)$ has therefore a sampling frequency of approximately $f_{s}=156 \mathrm{kHz}$. The $A_{D}(t)$ time series is computed from the area under the Doppler-shifted spectral peak.

An FFT is then once again used to calculate the average power spectrum from the $f_{D}(t)$ time series, up to the Nyquist frequency $f_{N y}=f_{s} / 2=78 \mathrm{kHz}$, which is well-suited for the study of GAMs at AUG.

An alternative approach to the sliding FFT is the Multiple Signal Classification (MUSIC) method [27], which has recently been proposed [12]. This algorithm presumes that the complex IQ signal $x(t)$ consists of $n_{f}$ coherent frequency components $f_{k}$ and an incoherent noise part $n(t): x(t)=\sum_{k=0}^{n_{f}} A_{k} \exp \left(-i 2 \pi f_{k} t\right)+n(t)$. By calculating 
the eigenvectors of the autocorrelation matrix via singular value decomposition, the contributions from signal and noise can be separated. The noise eigenvectors are then used to compute the pseudospectrum $P_{M U S I C}$, which shows a distinct peak at $f_{D}$. The main advantage of the MUSIC method is the possibility to calculate $f_{D}$ from a smaller window of the IQ signal, leading to a higher time resolution in $f_{D}(t)$, but at the cost of less stability.
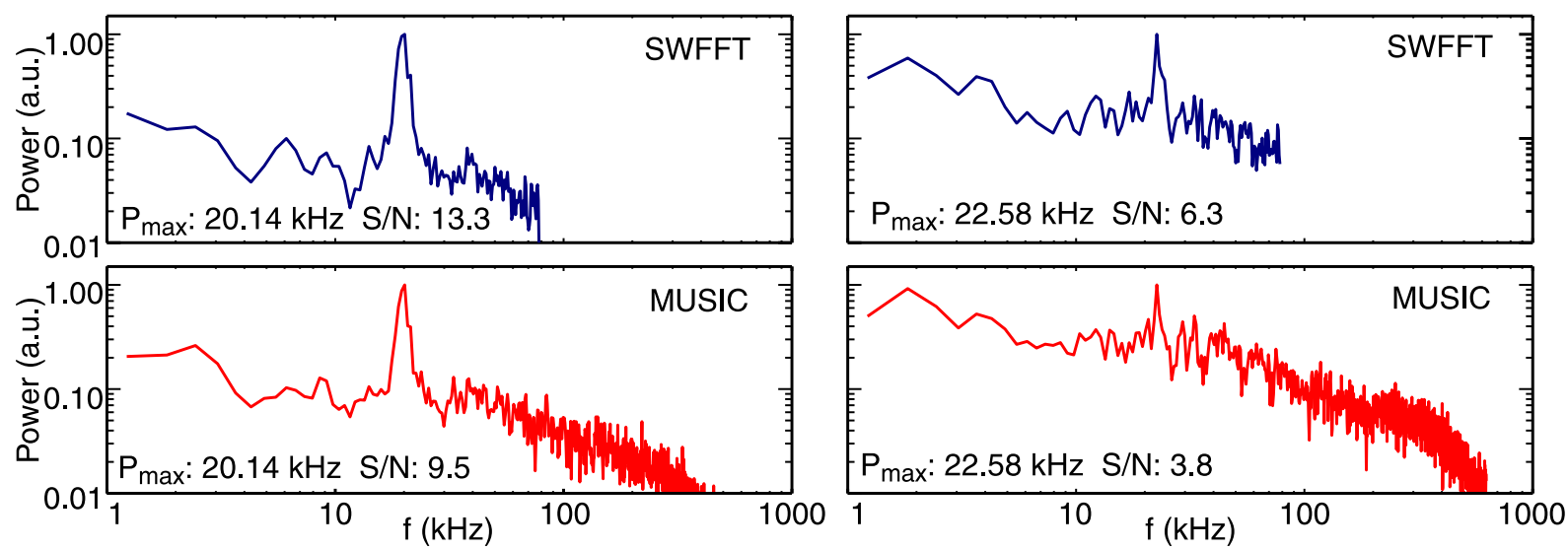

Figure 6. Comparison of sliding window FFT (SWFFT) and MUSIC methods for GAM detection. Left: Strong GAM (\#29650, $t=2.190-2.205 \mathrm{~s})$, Right: Weak GAM (\#29650, $t=2.220-2.235 \mathrm{~s}$ ). MUSIC algorithm parameters as defined in [12]: $n_{w s}=32, n_{\text {shift }}=16, n_{w}=6, n_{f}=1$ and $n_{\mathrm{FFT}}=8192$.

Figure 6 shows average power spectra computed via FFT from two exemplary experimental measurements, where GAMs of different intensity and frequency were found. The $f_{D}(t)$ signal was computed with both the sliding window FFT (SWFFT) method and with the MUSIC method for comparison. Both methods detect the GAM at the same frequency. The MUSIC algorithm uses multiple parameters for window size $\left(n_{w s}\right)$, overlap between windows $\left(n_{\text {shift }}\right)$, number of detectable frequencies $\left(n_{f}\right)$ and frequency resolution $\left(n_{\mathrm{FFT}}\right)$. Various combinations of these parameters were tested for GAM detection, and best results were generally obtained with $n_{w s}=32, n_{\text {shift }}=16$, $n_{w}=6, n_{f}=1$ and $n_{\mathrm{FFT}}=8192$. Generally, the SWFFT method is much faster to compute and manages to detect the GAM with slightly better signal to noise ratio. This is contrary to Tore Supra results where the MUSIC algorithm proved superior for GAM detection [12]. All experimental GAM measurements presented in this article have been obtained using the conventional SWFFT approach.

\section{GAM frequency results}

\subsection{Comparison with Winsor's scaling and Conway's heuristic model}

The prediction for the GAM frequency $f_{\mathrm{GAM}}$ from equation 1, derived by Winsor [5] from a fluid model for a circular plasma approximation, contains as parameters only the sound velocity $c_{s}$, the major radius $R_{0}$ and the safety factor $q$. For figure 7 , 


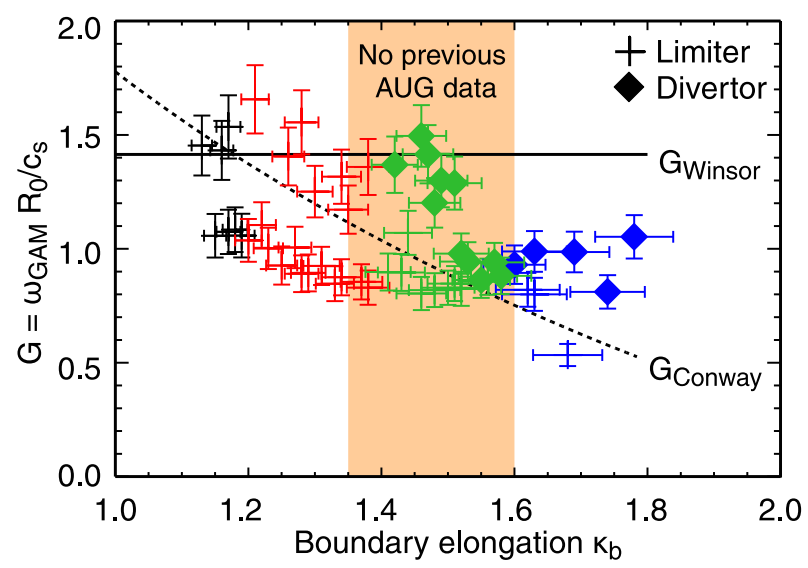

Figure 7. Scale factor $G=\omega_{\mathrm{GAM}} R_{0} / c_{s}$ as function of the plasma boundary elongation $\kappa_{b}$ for the whole data set with $3.5<q_{95}<5$, compared to the Conway and Winsor models.

the scale factor $G$ was determined from the shape-scan experiments, by measuring the GAM frequency and normalizing to $c_{s} / R_{0}$ (to remove the dependence of $f_{\mathrm{GAM}}$ on the local plasma temperature). Variations in $q$ were neglected for this figure. $G=\omega_{\mathrm{GAM}} R_{0} / c_{s}$ was then plotted over the plasma elongation at the corresponding time in the discharges. Highlighted is the area of intermediate plasma elongation, in which no previous experimental data was available.

The main sources of uncertainty for the scaling factor $G$ are the errors in the $T_{e}$ and $T_{i}$ (and therefore the sound velocity) and in the extraction of the GAM frequency from the spectrum. The errors in the temperatures consist mostly of contributions due to uncertainties in the ECE measurements and uncertainty in the measurement position due to the fitted density profile. These are estimated to be of the order of $15 \%$. The error in the GAM frequency is dictated by the frequency resolution due to the chosen FFT parameters and here is approximately $0.3 \mathrm{kHz}$, which is below $5 \%$ for all GAM measurements in the database. The uncertainty for the boundary elongation $\kappa_{b}$ is determined by the steepness of the $\kappa$ profile. For plasmas with low $\kappa_{b}$, the profile is relatively flat and small uncertainties in the radial coordinate only translate to minimal variation in $\kappa_{b}$. For elongated plasmas the $\kappa$ profile is steeper in the edge and an error of up to $5 \%$ is expected. A similar uncertainty is expected for variations in $q$.

As previously noted, Winsor's model does not take into account the plasma shape. In contrast, a clear trend can be observed towards lower GAM frequencies at higher elongation. The trend of decreasing frequency with elongation is captured in the heuristic model by Conway:

$$
\omega_{\mathrm{GAM}}=\frac{c_{s}}{R_{0}} 4 \pi\left(\frac{1}{1+\kappa_{b}}-\epsilon_{0}\right) .
$$

Here, $\epsilon_{0}=0.3$ is the inverse aspect ratio. However, simultaneously a systematic difference between GAMs in limiter discharges (crosses) and in divertor discharges (diamonds) can be seen. 


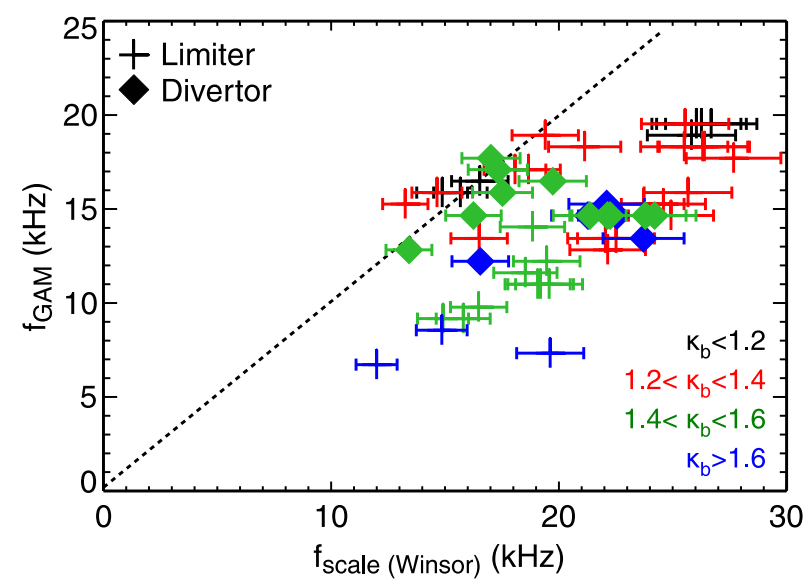

Figure 8. Comparison of the experimental results $\left(f_{\mathrm{GAM}}\right)$ with Winsor's scaling law $\left(f_{\text {scale }}\right)$. All points refer to GAMs in a range of radial positions $0.91<\rho_{\text {pol }}<0.99$ and safety factor $3.5<q_{95}<5$. The degree of elongation is indicated by colour.

Figure 8 shows the experimentally observed GAM frequency as a function of the frequency predicted by Winsor's scaling. The uncertainty in $f_{\text {scale }}$ is due to the errors in the temperatures, as described above. The error in $f_{\mathrm{GAM}}$ is not pictured as it is comparable to the symbol size. As already seen in figure 7, Winsor's scaling generally overestimates $f_{\text {GAM }}$. For a number of points at close to circular plasmas, which meet the assumption made in the model, the prediction agrees with the data, but especially for high $\kappa_{b}$ the discrepancy becomes large. This confirms earlier AUG results [14].

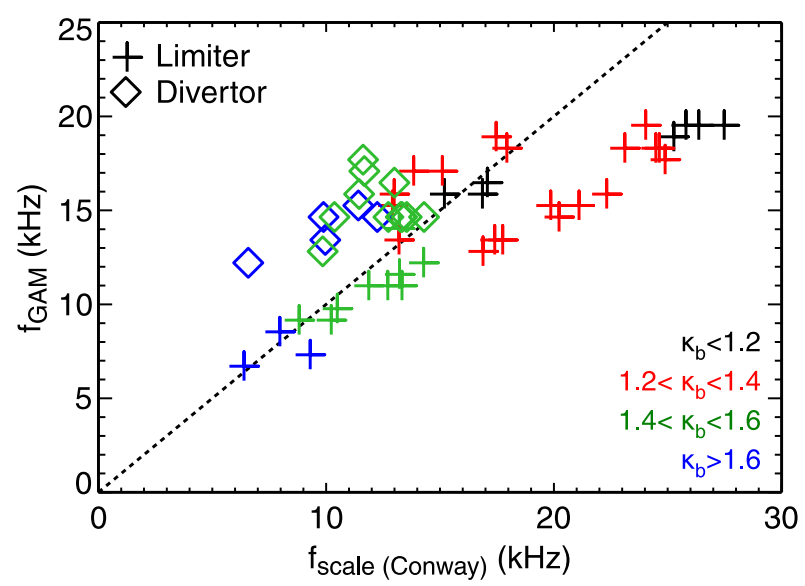

Figure 9. Comparison of the experimental results $f_{\mathrm{GAM}}$ with Conway's scaling law $f_{\text {scale. }}$

Figure 9 shows a comparison with the heuristic Conway scaling, which accounts for the effect of the plasma elongation. For better visibility there are no error bars included in this and the following figures. As the uncertainties in the temperatures are the strongest influence on the errors in all frequency scalings, the error bars in figure 8 may be used as a reference. 
This empirical model was found to give good agreement for GAMs in the edge of elongated plasmas, but does not reduce to Winsor's scaling for $\kappa_{b} \rightarrow 1$. The model also does not include the influence of $q$, as its effect on the experimental GAM frequency was not found to be strong [14].

In comparison to Winsor's scaling, the Conway model yields better agreement to the present data. However, there are still systematic deviations from the experimental data: in limiter discharges, GAMs closer towards the plasma center, i.e. in regions of higher plasma temperature, are found to be at a lower frequency than predicted. For limiter GAMs in the plasma edge region a good agreement is found. For GAMs in divertor discharges the experimentally measured frequency appears to be generally higher than predicted.

\subsection{Comparison with Angelino's fluid model}

In order to establish an analytical relation between GAM properties and the plasma geometry, in 2008 Angelino et al. derived a prediction for the GAM frequency from a fluid model [19]. The derivation takes the geometry into account through the local elongation $\kappa[28]$ :

$$
\omega_{\mathrm{GAM}}=\frac{v_{T_{i}}}{R_{0}} \sqrt{\left(\tau+\gamma_{i}\right)\left[\frac{8}{3-2 \kappa+3 \kappa^{2}}+\frac{1}{q^{2}}\right]}
$$

Here, $\tau=T_{e} / T_{i}, \gamma_{i}=1$ and $v_{T_{i}}=\sqrt{T_{i} / m_{i}}$. Angelino also uses the local elongation $\kappa$ instead of boundary elongation $\kappa_{b}$. For the comparison, $\kappa$ is obtained from the CLISTE equilibrium code [29]. For $\kappa \rightarrow 1$, the formula reduces to Winsor's scaling (equation 1).

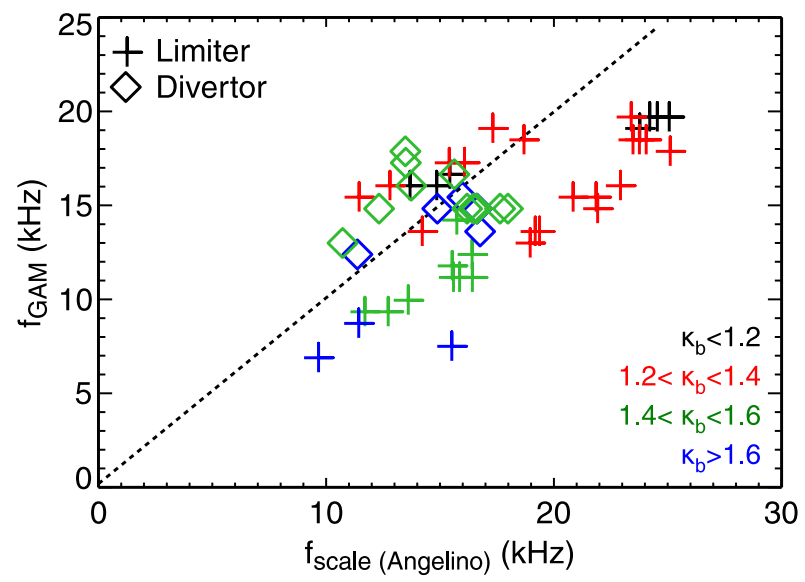

Figure 10. Comparison of the experimental results $\left(f_{\mathrm{GAM}}\right)$ with Angelino's scaling law $\left(f_{\text {scale }}\right)$.

The comparison of measured GAM frequencies to Angelino's scaling are shown in figure 10. The agreement is similar to that obtained with Conway's heuristic model. In general, the scaling fits the data much better than Winsor's simplified fluid model, but 
there are large discrepancies for limiter GAMs at low $\kappa$, towards the plasma core. The predicted GAM frequency lies above the measurements. However, Angelino's scaling appears to give a better fit for GAMs in divertor plasmas.

\subsection{Comparison with Gao's gyrokinetic model}

A more involved approach based on an electrostatic gyrokinetic model and incorporating the effects of finite orbit drift width (ODW) was derived by Gao [30, 31, 17] between 2009 and 2011. In contrast to Angelino's approach, Gao includes a larger number of geometric parameters. Non-linear effects are not included. In the small ODW limit the GAM frequency is given by

$$
\begin{aligned}
\omega_{\mathrm{GAM}}= & \frac{v_{T_{i}}}{R_{0}} \sqrt{\left(\frac{7}{4}+\tau\right)\left(\frac{2}{\kappa^{2}+1}\right)\left(1-\frac{s_{\kappa}}{2} \frac{7+2 \tau}{7+4 \tau}\right)}\left[1-\epsilon^{2} \frac{9 \kappa^{2}+3}{8 \kappa^{2}+8}\right. \\
& \left.-\Delta^{\prime 2} \frac{\kappa^{2}}{4 \kappa^{2}+4}+\epsilon \Delta^{\prime} \frac{4 \kappa^{2}+1}{4 \kappa^{2}+4}+\frac{\left(23+16 \tau+4 \tau^{2}\right)\left(\kappa^{2}+1\right)}{2 q^{2}(7+4 \tau)^{2}}\right],
\end{aligned}
$$

with $\tau=T_{e} / T_{i}$, inverse aspect ratio $\epsilon=r / R_{0}$, Shafranov shift gradient $\Delta^{\prime}$ and the radial derivative of the elongation $s_{\kappa} \approx(\kappa-1) / \kappa$. Again, the local elongation $\kappa$ is used. Typical values for $\Delta^{\prime}$ in the region of GAM activity are between -0.4 and -0.2. Therefore the contribution of the Shafranov shift gradient towards the GAM frequency is of a similar order of magnitude as the inverse aspect ratio, both of which decrease $\omega_{\mathrm{GAM}}$ by about $10 \%$ for typical values of $\kappa$.

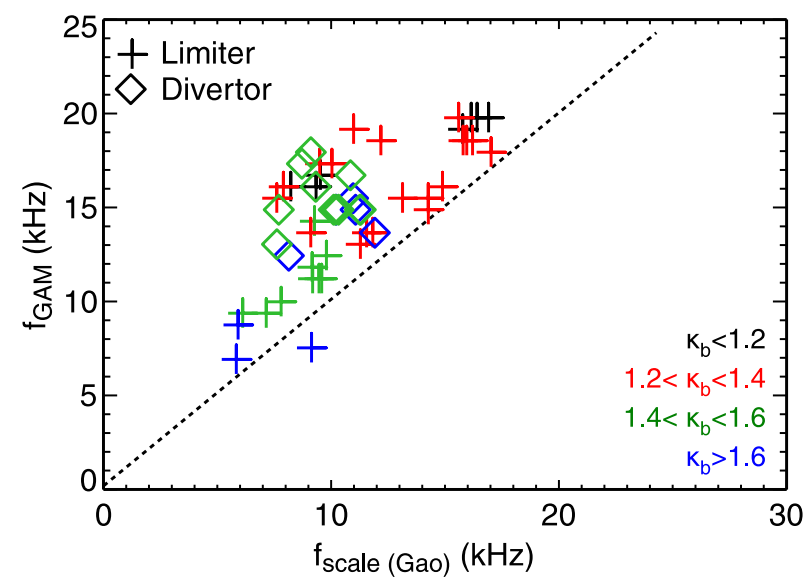

Figure 11. Comparison of the experimental results $\left(f_{\mathrm{GAM}}\right)$ with the Gao scaling law $\left(f_{\text {scale }}\right)$.

The comparison of the experimental data with the Gao scaling in figure 11 shows some improvements. While in general the experimental frequency lies above the theoretical prediction, the discrepancy between edge and core GAMs in limiter discharges mostly disappears, and the deviation from divertor discharges is less severe. In general, Gao's scaling exhibits the least scatter between various radial regions or plasma geometries and seems the closest approach so far to presenting a unified picture. 


\subsection{Non-linear effects}

Due to the linear derivation of Gao's equation, the predicted frequency can be understood to give only an estimate, whereas non-linear effects may also play a role. The results show a measured GAM frequency always shifted upward with respect to the linear theoretical prediction. This seems consistent with the nonlinear gyrokinetic prediction, according to which the nonlinear frequency shift is always positive [32, 33], whereas nonlinear MHD predictions give both upshifts and downshifts depending on the phase delay between the nonlinear force and the GAM oscillation [34]. A detailed analysis of the nonlinear frequency modification is outside the scope of this paper, and will be addressed in a future paper.

\subsection{Electromagnetic effects}

A further factor that should be considered is the influence of electromagnetic effects. While the investigated models are based on electrostatic approaches, recent modelling [35] suggests that plasma $\beta$ can lead to lowered GAM frequencies. This has been observed in an ASDEX Upgrade discharge, where a $100 \%$ increase in $\beta$ is observed with ECR heating, the GAM frequency scaling is about $10 \%$ lower than expected. For the discharges used in this database, however, $\beta$ only changes by less than $5 \%$ in limiter discharges in all cases, while in a few extreme divertor cases at low elongation $\beta$ increased only by a maximum of $50 \%$. Therefore, electromagnetic effects are not expected to play a significant role for the majority of the database, and may decrease the frequency for a limited number of low- $\kappa$ divertor GAMs, but within the error bars given by the temperature measurements. As electromagnetic models are only recently being investigated, further comparison is beyond the scope of this article.

\section{GAM amplitude results}

The amplitude of the GAM is obtained from the power spectrum of the $f_{D}(t)$ time series. The GAM peak at frequency $f_{\text {GAM }}$ is typically distinct from the background, as seen in the highlighted area of figure 5 (bottom). The amplitude is calculated as the peak-to-peak velocity by integrating over the GAM spectral peak [7]:

$$
A_{\mathrm{GAM}}=\frac{4 \pi}{k_{\perp}} \sqrt{\sum_{f_{1}}^{f_{2}} S\left(f_{D}\right) \frac{4}{1.5}},
$$

where $f_{1}, f_{2} \approx f_{\mathrm{GAM}} \pm 0.6 \mathrm{kHz}$. The factor $4 / 1.5$ accounts for the Hanning bellwindow that is applied to minimize spectral leakage. The perpendicular wavenumber $k_{\perp}$ is calculated using beam tracing and is typically of the order of 9-10 $\mathrm{cm}^{-1}$. As shown in figures 12 and 13, the GAM amplitudes for this set of discharges fall in the range of $0.2-1.0 \mathrm{~km} / \mathrm{s}$. For plasma conditions where GAMs are usually measured, i.e. the edge region of L-mode plasmas, the mean perpendicular plasma velocity is in the range of 
$2-5 \mathrm{~km} / \mathrm{s}[22]$. Hence the GAM can cause a perturbation of the plasma velocity by up to $50 \%$. The ratio between GAM amplitude and frequency is used to estimate the poloidal displacement caused by the GAM, and is of the order $A_{\mathrm{GAM}} / \omega_{\mathrm{GAM}} \approx 1 \mathrm{~cm}$, which is comparable to typical values of the poloidal turbulence correlation length in the edge (see $[36,37]$ and references therein). The radial edge turbulence correlation length is of similar size and is also comparable to the observed radial extent of GAMs in AUG plasmas [14]. The dataset is formed from values taken at the GAM radial maximum in each discharge condition.

The stationary GAM amplitude results from a balance between the non-linear drive from the gradient-driven turbulence, damping due to collisional and collisionless processes and the energy transfer between various scales. Previously, the local temperature gradient $\nabla T_{e}$, normalized by $\sqrt{\kappa_{b}}$, was used as a proxy for the turbulence drive in ASDEX Upgrade [7]. In this new dataset, the factor $\nabla T_{e} / \sqrt{\kappa_{b}}$ is roughly constant in the range of $5-7 \mathrm{keV} / \mathrm{m}$. In addition, variations in the turbulence level have also been measured directly using normal incidence reflectometers. Here, the turbulence levels across the dataset are of similar orders of magnitude. Any variations may be attributed to the radial dependence of the turbulence. Additionally, the turbulence level is subject to the dynamics of energy transport processes. The energy transfer rate between the background turbulence and the GAM is an unknown factor which is not addressed further in this work. The primary focus here is placed on the importance of the GAM damping terms.

\subsection{Dependence on $\kappa_{b}$ and $q$}

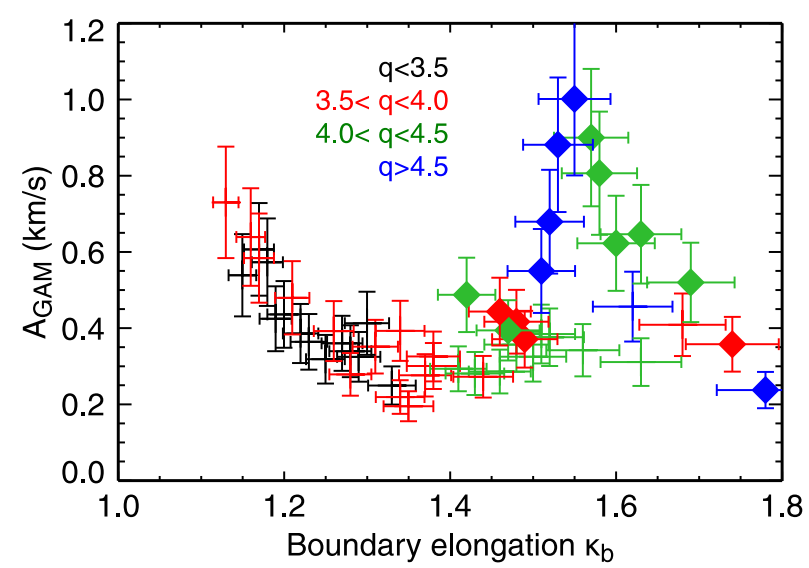

Figure 12. GAM amplitude against boundary elongation $\kappa_{b}$, colour-coded for varying local safety factor $q$.

Figure 12 shows GAM amplitudes for the whole dataset against the plasma boundary elongation $\kappa_{b}$, colour-coded for ranges in local safety factor $q$. Figure 13 is the counterpart, showing GAM amplitude against $q$, with $\kappa_{b}$ ranges marked by the colour. The error in the GAM amplitude is influenced by the uncertainties of $k_{\perp}$ and 


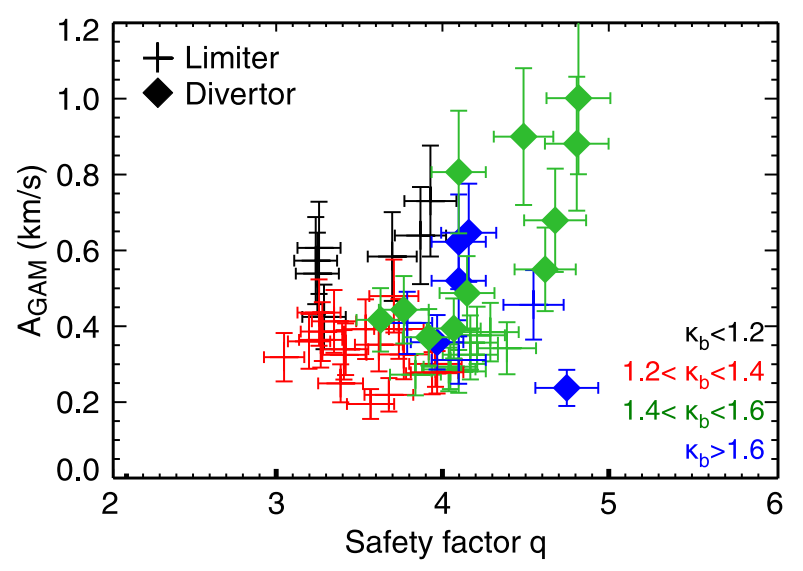

Figure 13. GAM amplitude against local safety factor $q$, colour-coded for varying boundary $\kappa_{b}$.

the integration over the GAM peak. It is approximated as $20 \%$. The errors in $\kappa_{b}$ and $q$ are estimated as discussed in section 4.1.

For limiter discharges the results in figure 12 are similar to earlier ASDEX Upgrade experiments [7] with the added benefit of a large number of points in a previously unavailable region for $\kappa_{b}$. There is a clear inverse dependence of $A_{\mathrm{GAM}}$ on the elongation $\kappa_{b}$, and only a weak direct dependance on local $q$ evident. For $\kappa_{b}>1.4$ the amplitude tends to become insensitive to elongation. There is also a trend for higher $\kappa_{b}$ to be accompanied by higher $q$.

For the case of divertor plasmas the effect of the X-point and strong shaping is more evident with discharges generally having higher $q$. The lowest GAM amplitudes in divertor plasmas are either found at low $q$, or at very high $\kappa_{b}$. Very high $q$ raises $A_{\mathrm{GAM}}$, while for moderate $q(3.5-4.5)$, the impact of $\kappa_{b}$ is seen again. For low to moderate $q$ $(3-4), A_{\mathrm{GAM}}$ approaches the limiter values.

The dependence of $A_{\mathrm{GAM}}$ on $q$ is more clearly seen in figure 13. Low-amplitude GAMs can appear regardless of the local $q, \kappa_{b}$ or plasma configuration. Although, as shown in figure 12 , there is a tendency to higher $A_{\mathrm{GAM}}$ at lower $\kappa_{b}$ for a fixed $q$. There is also a clear trend of high $A_{\mathrm{GAM}}$ at higher $q$ in divertor configuration. As a general observation the influence of $\kappa_{b}$ shows a clear trend for limiter data, while the influence of $q$ appears more important for divertor plasmas.

\subsection{Dependence on damping}

Previously there has been a focus on collisionless Landau damping, which is often approximated as $\gamma \propto \omega_{\text {GAM }} \exp \left(-q^{2}\right)[38,19,7]$. Gao has more recently derived a more extensive formula from a gyrokinetic approach in the limit of negligible GAM radial wavenumber $k_{r} \rightarrow 0$ [39]:

$$
\gamma_{\text {c.less }}=-\frac{\pi^{1 / 2}}{2} \frac{v_{T_{i}}}{R} \frac{\left(R \omega_{\mathrm{GAM}} / v_{T_{i}}\right)^{6}}{7 / 4+\tau} q^{5} \exp \left[-\left(\frac{q R \omega_{\mathrm{GAM}}}{v_{T_{i}}}\right)^{2}\right]
$$


This formula gives a monotonically decreasing $\gamma$ in the experimental range $3<$ $q<5$, which appears to be consistent with the results of figure 13 showing the GAM amplitude increasing with $q$.

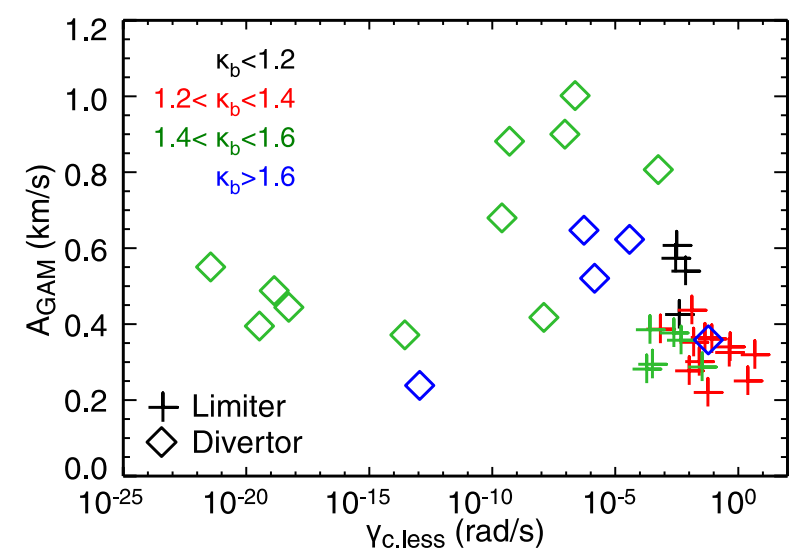

Figure 14. GAM amplitude against collisionless damping rate $\gamma_{\text {c.less }}$ in the limit $k_{r} \rightarrow 0$ (equation 6).

Figure 14 shows the measured GAM amplitude $A_{\mathrm{GAM}}$ as a function of the collisionless damping rate $\gamma_{\text {c.less }}$, according to equation 6 . There is a very large variation in $\gamma_{\text {cless }}$, over 25 orders of magnitude. Due to the large scatter of $A_{\mathrm{GAM}}$, there is no clear dependence between damping rate and GAM amplitude, contrary to what would be expected if collisionless damping was the dominant process. While the lowest $A_{\mathrm{GAM}}$ are found at high $\gamma$, there is no straightforward trend for the limiter data, and the divertor $A_{\mathrm{GAM}}$ even tend to be larger at higher collisionless damping rates. If values below $10^{-10} \mathrm{~Hz}$ are ignored there is perhaps a trend of decreasing $A_{\mathrm{GAM}}$ with rising $\gamma_{\text {cless }}$. Nevertheless, the plasma elongation appears to have an influence on the collisionless damping rate, as most GAMs with $\kappa_{b}>1.4$ have significantly lower $\gamma$. This is due to the factor $\omega_{\mathrm{GAM}} R / v_{T_{i}}$ in the exponential, which will decrease for elongated plasmas (cf. figure 7 ). Further, the damping rates for divertor plasmas are much lower than those for limiter plasmas, with the exception of only a few points.

Recent theoretical work ([40] and references therein) and simulations [41], however, suggest that finite orbit width effects, i.e. $k_{r}>0$, may play a significant role for the collisionless damping rate in the case of $q>2$. Sugama and Watanabe used a gyrokinetic approach to derive an expression that includes the finite orbit width effects [18, 42]:

$$
\begin{aligned}
\gamma_{\mathrm{SW}}= & -\frac{\pi^{1 / 2}}{2} \frac{v_{T_{i}} q}{R_{0}}\left[1+\frac{2\left(23 / 4+4 \tau+\tau^{2}\right)}{q^{2}(7 / 2+2 \tau)^{2}}\right]^{-1} \\
& \times\left[\exp \left(-\hat{\omega}_{\mathrm{G}}^{2}\right)\left\{\hat{\omega}_{\mathrm{G}}^{4}+(1+2 \tau) \hat{\omega}_{\mathrm{G}}^{2}\right\}+\frac{1}{4}\left(\frac{k_{r} v_{T_{i}} q}{\Omega_{i}}\right)^{2}\right. \\
& \left.\times \exp \left(-\hat{\omega}_{\mathrm{G}}^{2} / 4\right)\left\{\frac{\hat{\omega}_{\mathrm{G}}^{6}}{128}+\frac{1+\tau}{16} \hat{\omega}_{\mathrm{G}}^{4}+\left(\frac{3}{8}+\frac{7}{16} \tau+\frac{5}{32} \tau^{2}\right) \hat{\omega}_{\mathrm{G}}^{2}\right\}\right]
\end{aligned}
$$


Here, $\hat{\omega}_{\mathrm{G}}=R_{0} q \omega_{\mathrm{GAM}} / v_{T_{i}}$ and $\Omega_{i}=e B / m_{i}$. For all calculations of the damping rate, the experimentally measured GAM frequency $\omega_{\text {GAM }}$ was used. The impact of the finite orbit width effect is very sensitive to the radial wavenumber $k_{r}$ of the GAM. In AUG the radial wavelength $\lambda_{r}$ of the GAM is obtained from radial cross-correlation measurements [43] to be of the order of $4-5 \mathrm{~cm}$, i.e. typically twice the zonal width of the GAMs. This estimate is consistent with previous width and spacing of the GAM frequency plateaus [14]. A $\lambda_{r}$ of this magnitude gives a corresponding $k_{r}$ of approximately $150 \mathrm{~m}^{-1}$, which should be considered as an upper boundary. $k_{r}$ of this magnitude have also been measured in DIII-D [44].

Qiu et al. [16] note that equation 7 is only valid for $k_{r} v_{T_{i}} q^{2} / \Omega_{i} \ll 1$, which is not the case here. In the limit of large ODW the dominant resonant mechanism changes from low order harmonic transit resonance to high order harmonic resonance, and the damping rate becomes independent of $q$. Gao derives the following equation for the collisionless damping rate in the large ODW limit from a gyrokinetic approach, which includes various effects, such as the plasma geometry [31, 17]:

$$
\begin{aligned}
\gamma_{\text {LODW }}= & -\frac{4 \kappa^{2} \sqrt{7 / 4+\tau}}{\hat{k}^{2}\left(\kappa^{2}+1\right)^{3 / 2}} \frac{v_{T_{i}}}{R_{0}}\left(1+\frac{2 \kappa^{2}+5}{4 \kappa^{2}+4} s_{\kappa}-\frac{27 \kappa^{2}+9}{8 \kappa^{2}+8} \epsilon^{2}-\frac{7 \kappa^{2}+4}{4 \kappa^{2}+4} \Delta^{\prime 2}\right. \\
& \left.+\frac{9 \kappa^{2}}{4 \kappa^{2}+4} \epsilon \Delta^{\prime}\right) \times \exp \left[-\frac{\sqrt{7 / 4+\tau}}{\hat{k}} \sqrt{\frac{2 \kappa^{2}}{\kappa^{2}+1}}\left(1+\frac{3 \kappa^{2}+4}{4 \kappa^{2}+4} s_{\kappa}\right.\right. \\
& \left.\left.-\frac{9 \kappa^{2}+3}{8 \kappa^{2}+8} \epsilon^{2}-\frac{3 \kappa^{2}+2}{4 \kappa^{2}+4} \Delta^{\prime 2}+\frac{4 \kappa^{2}+1}{4 \kappa^{2}+4} \epsilon \Delta^{\prime}\right)\right]
\end{aligned}
$$

Figure 15 shows a comparison of the three collisionless damping values for the whole database, plotted against the normalized poloidal flux radius $\rho_{\text {pol }}$, with $\gamma_{\text {cless }}$ in red from equation $6, \gamma_{\mathrm{SW}}$ in green from equation 7 and $\gamma_{\mathrm{LODW}}$ in blue from equation 8 . For the calculation of $\gamma_{\mathrm{SW}}$ and $\gamma_{\mathrm{LODW}}$, a constant estimate of $k_{r}=150 \mathrm{~m}^{-1}$ is used. While for selected cases, $k_{r}$ has been measured between 75 and $150 \mathrm{~m}^{-1}$, accurate measurements for each GAM in the database do not exist, and the calculated damping rates should be taken as an upper boundary. Including the low $k_{r}$ effects raises $\gamma_{\text {cless }}$ by 4 to 12 orders of magnitude, depending on radius and $q$. In the large ODW limit, the damping rate $\gamma_{\text {LODW }}$ lies approximately 2 to 3 orders of magnitude below $\gamma_{\mathrm{SW}}$, but still significantly above the $\gamma_{\text {c.less }}\left(k_{r} \rightarrow 0\right)$ value.

The effect of collisional damping should also be taken into account. As recently noted by Gao [40], collisional damping has often been ignored as it was not thought to play a large role in the plasma core. But, as GAMs are observed in the plasma edge region, the collisional damping may in fact play the dominant role. A first estimation of the collisional damping rate for GAMs was given by Novakovskii et al. as $\gamma=-4 / 7 \nu_{i}$ (the original publication mistakenly included a $q$ in the denominator) $[45,3]$. The ion collisionality in a deuterium plasma is defined as $\nu_{i}=4.80 \times 10^{-2} \sqrt{1 / 2} n_{e} \ln \Lambda T_{i}^{-3 / 2} \mathrm{~s}^{-1}$, $n_{e}$ is in $\mathrm{m}^{-3}, T_{i}$ in $\mathrm{eV}$ and the Coulomb logarithm is $\ln \Lambda \approx 17$. 

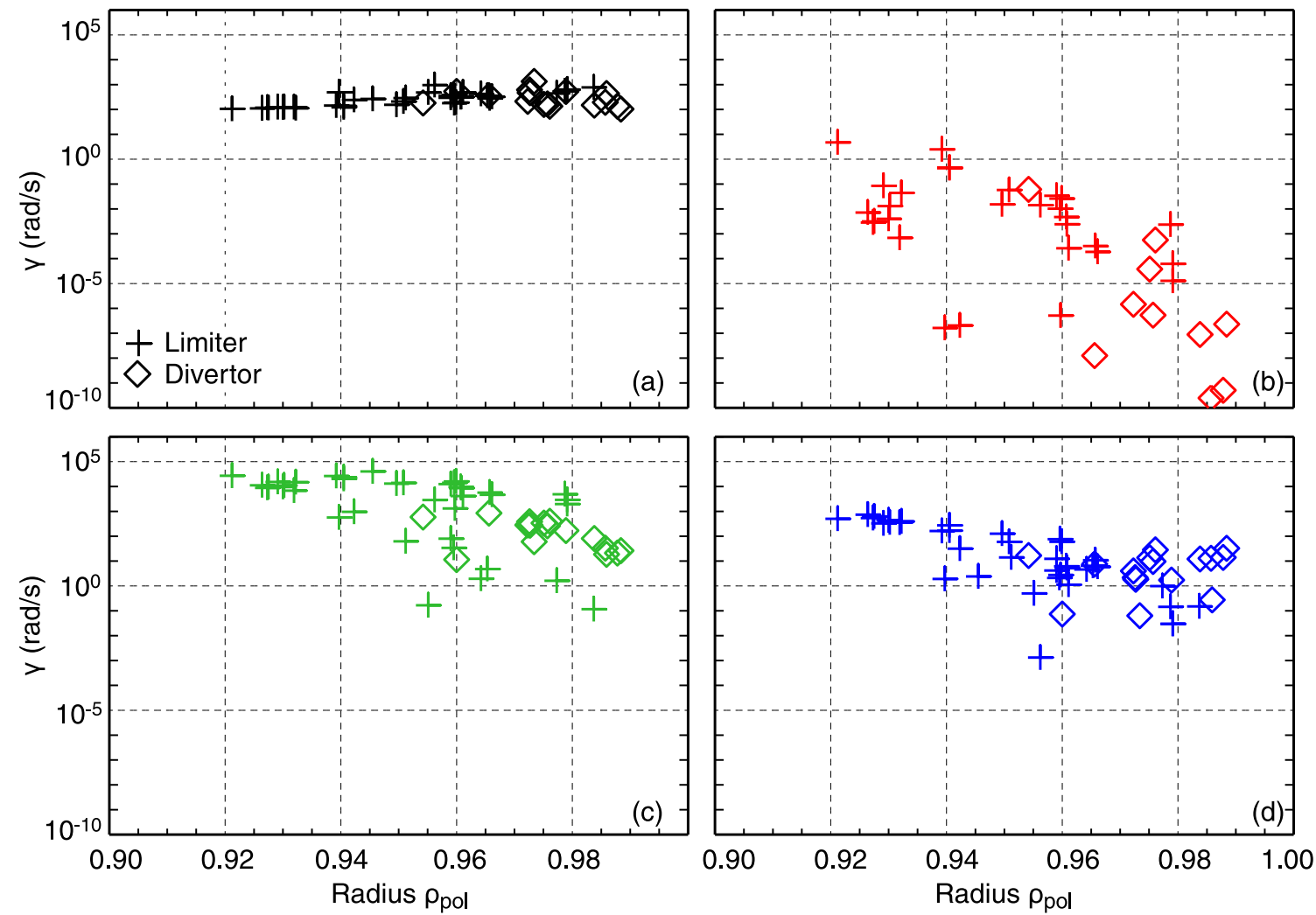

Figure 15. Comparison of damping rates against normalized poloidal flux radius $\rho_{\text {pol }}$. (a) Collisional damping rate (black). (b) Collisionless damping for $k_{r} \rightarrow 0$ (red). (c) Collisionless damping with finite ODW corrections (green). (d) Collisionless damping in the large ODW limit (blue). A constant value of $k_{r}=150 \mathrm{~m}^{-1}$ is used.

Using a gyrokinetic model, Gao derived the following equation for the collisional damping rate [40]:

$$
\left(\frac{7}{4}+\tau\right) \frac{q R}{v_{T_{i}}} \gamma+\frac{3}{8} \frac{q R}{v_{T_{i}}} \nu_{i}+\frac{q R^{3}}{v_{T_{i}}^{3}} \gamma\left(4 \gamma^{2}+4 \gamma \nu_{i}+\nu_{i}^{2}\right)=0
$$

In the case of $\nu_{i} q R / v_{T_{i}} \ll 1$ and $\gamma q R / v_{T_{i}} \ll 1$, the formula simplifies to

$$
\gamma_{\text {coll }}=-\frac{3}{14+8 \tau} \nu_{i}
$$

Numerical calculations confirm that all the AUG experimental GAM measurements satisfy this condition. Note, unlike equation 6 , there is no implicit $\kappa$-dependence here.

Figure 15(a) shows the collisional damping $\gamma_{\text {coll }}$ from equation 10 in black. A comparison of the various damping rates indicates that, if ODW effects are neglected, the collisional damping rate is orders of magnitude above the collisionless damping rate $\gamma_{\text {c.less }}$ - an observation similarly noted recently in Tore Supra [15]. However, when the contribution of $k_{r}$ is taken into account the collisionless damping rate can become dominant in limiter low $q$ conditions, while at high $q$ limiter and divertor edge conditions the collisional term may still dominate or be comparable to the collisionless damping rate. 

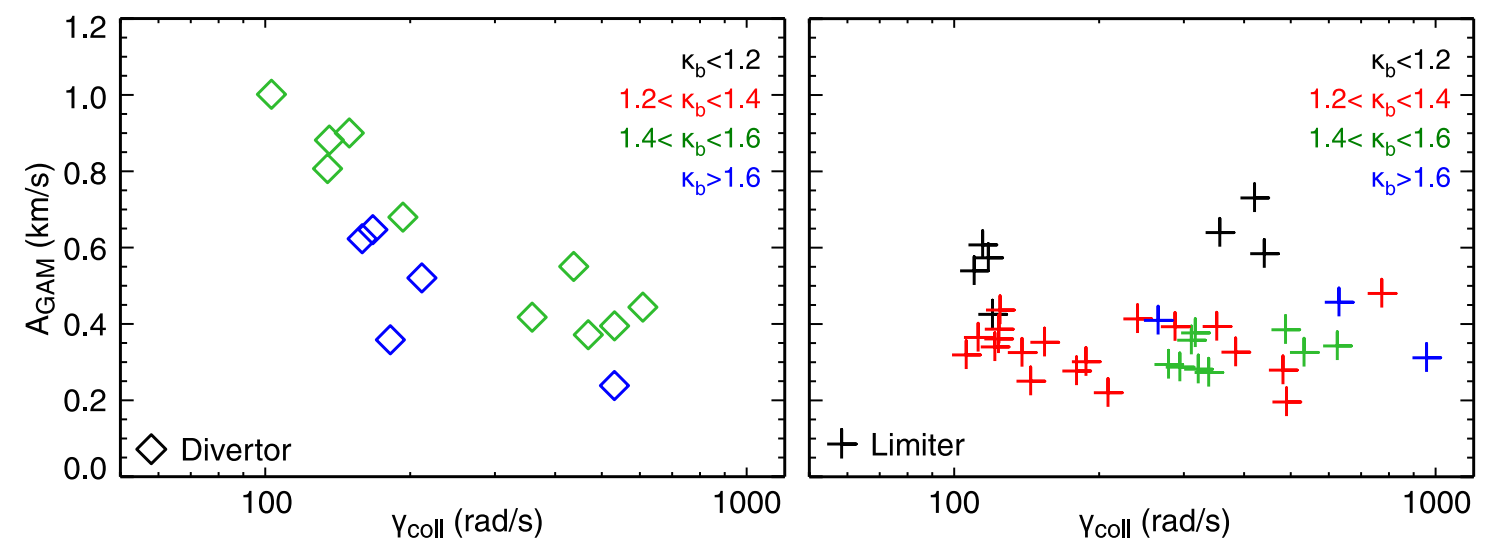

Figure 16. Scaling of GAM amplitude against collisional damping rate $\gamma_{\text {coll }}$. Left: Divertor plasmas. Right: Limiter plasmas.

Experimentally, the variation of $A_{\mathrm{GAM}}$ with collisional damping is different between limiter and divertor configurations. Figure 16 shows the GAM amplitude as a function of $\gamma_{\text {coll }}$ from equation 10 for divertor and limiter plasmas. In the divertor case there is a much clearer trend of decreasing $A_{\mathrm{GAM}}$ with rising $\gamma_{\text {coll }}$. In the limiter case, there is more spread in the data, and for each subset of measurements at similar $\kappa_{b}$ there is almost no variation with $\gamma_{\text {coll }}$. The collisional damping appears to have the effect of setting an upper boundary on the GAM amplitude, particularly for divertor plasmas. In the case of limiter plasmas the GAM amplitude seems to be determined by the boundary elongation, as seen in figure 12 .

\section{Discussion and summary}

This article presents experimental results from Doppler reflectometry measurements on a number of shape-scan experiments in order to study the influence of plasma geometry on the properties of the geodesic acoustic mode. The MUSIC algorithm is tested as an alternative to the conventional sliding FFT approach for GAM measurements, but unlike results from Tore Supra [12] it is not shown to improve GAM detection at ASDEX Upgrade. The measurements are compared to heuristic and various theorybased models. It is confirmed that the GAM frequency prediction by Winsor's simplified fluid model shows inaccuracies, as it generally overestimates the GAM frequency, with strong deviations for edge GAMs in elongated tokamak experiments. A comparison with Conway's heuristic model, which was based on a set of low- and high-elongation discharges, yields better agreement, even with the inclusion of new experimental points from limiter discharges at medium plasma elongation. However, discrepancies are still found, especially for lower- $\kappa$ limiter discharges, where the GAM frequency is overestimated. Also GAMs in divertor discharges seem to show a systematic disagreement with the empirical formula. More involved theory-based models attempt to account for the observed effects: a fluid model derived by Angelino, which incorporates 
the effect of plasma elongation $\kappa$, yields some improvement on Winsor's scaling. However, large discrepancies are found for low- $\kappa$ limiter plasmas. The overall agreement with the experimental data is still better for the heuristic model. Angelino's model is nevertheless useful as an estimate of the $\omega_{\mathrm{GAM}^{-}}$-dependence on $\kappa$ based on theory. Gao's gyrokinetic-based scaling is the most rigorous model in its inclusion of plasma geometry parameters such as $\epsilon, s_{\kappa}$ and $\Delta^{\prime}$. It appears to give a lower boundary prediction of the GAM frequency. The systematic underestimation of $\omega_{\mathrm{GAM}}$ suggests that non-linear effects may play a significant role and should be considered for an accurate prediction of the GAM frequency, however the possibility of non-linear frequency downshift must also be considered.

The presence of GAMs has been confirmed in many plasma devices (see the review by Fujisawa [8] and the references therein) and an overestimation of GAM frequency by the Winsor model has been confirmed on a number of experiments, among others at ASDEX Upgrade [11], DIII-D [6, 44], MAST [46] and Globus-M [47]. The thorough comparison of circular Tore Supre measurements and simulations against various models [15] points to several important influences on the GAM frequency. While the divertor geometry allows for more flexible shaping studies at ASDEX Upgrade, especially in terms of plasma boundary elongation $\kappa_{b}$, the Tore Supra results also stress the importance of impurities, which may account for discrepancies of up to $10 \%$ in case of high effective charge $Z_{\text {eff }}>2.5$. In typical ASDEX Upgrade edge scenarios, $Z_{\text {eff }}$ is not expected to exceed 1.5, therefore impurities should only provide a small correction. In the comparison of ASDEX Upgrade data with the gyrokinetic model, it is shown that other shaping terms which can be varied only in a limited range, such as the inverse aspect ratio $\epsilon$ and the Shafranov shift gradient $\Delta^{\prime}$ may also play a non-negligible role. Recent results from the spherical Globus-M tokamak only show good agreement with the GAM frequency predicted by the gyrokinetic scaling, highlighting the importance of the terms including the inverse aspect ratio $\epsilon$. There are still more parameters that are expected to influence the GAM frequency, but which are not always easily varied and studied. Pressure anisotropy, for example, is expected to impact the GAM frequency [48], but was shown to have little effect at Tore Supra [15]. Toroidal rotation is expected to provide an upshift of the GAM frequency $[49,50]$ but the magnitude of this effect might be small due to little external momentum input in the plasma discharges investigated here. A relatively strong influence on the GAM frequency, which has not to date been studied in detail, can be expected from variations in the isotope used during a discharge. First ASDEX Upgrade results show an increase of the GAM frequency by $30-40 \%$ when the plasma fuelling is changed from deuterium to hydrogen, which matches the change of $c_{s} \propto m_{i}^{-0.5}$.

The behaviour of the GAM amplitude is also studied in relation to the plasma parameters $\kappa$ and $q$. The GAM amplitude is seen to decrease in limiter plasmas with increasing elongation. Low- $q$ GAMs from divertor plasmas exhibit similar amplitudes, whereas the GAM amplitude is found to increase significantly at higher safety factor. The GAM amplitude scaling has been less often studied than the GAM frequency 
scaling in recent research, with little detailed investigation of the effects of plasma configuration and geometry [8]. Prior results from DIII-D suggest that a large safety factor $q$ might lead to higher GAM amplitudes due to reduced collisionless damping [6], while first ASDEX Upgrade results exhibit different observations for limiter and divertor plasmas [7]. In the limiter cases the boundary elongation has a clear effect on the GAM amplitude, but not in the divertor case. Here, the role of the X-point in the divertor configuration requires further investigation.

In this article the roles of collisionless Landau damping and collisional damping were investigated in more detail for a dataset with roughly similar turbulence drive $\nabla T_{e} / \sqrt{\kappa_{b}}$. In the absence of $k_{r}$ effects collisional damping clearly dominates over collisionless damping towards the outer plasma edge region. More towards the core region, however, the rising trend of the collisionless damping may cause $\gamma_{\text {cless }}$ to become dominant. Tore Supra results similarly predict a stronger influence of collisional damping in the relevant plasma edge region [15]. With inclusion of finite orbit drift width effects, the collisionless damping is increased overall (green and blue points in figure 15). The green points can be considered an upper boundary to the collisionless damping rates, based of the current measured $k_{r}$ estimates. A lower $k_{r}$ would reduce $\gamma_{\mathrm{SW}}$ towards the original $\gamma_{\text {c.less }}$ (red points). The large ODW limit values $\gamma_{\text {LODW }}$ are likewise reduced with decreasing $k_{r}$. Within this picture, the collisional damping would play a larger role for the divertor high $q$ cases in the plasma edge, while collisional and collisionless damping may play equal roles for limiter low $q$ cases towards the plasma core. This switch in damping dependency may go towards explaining the different behaviours observed in figure 16 for the limiter and divertor configurations.

Comparisons to numerical modelling for ASDEX Upgrade plasmas are in progress, but first results from the gyrokinetic code NEMORB for a circular plasma geometry [41] already show a good agreement for the GAM frequency and damping rate with the gyrokinetic based model of Sugama and Watanabe.

\section{Acknowledgments}

The authors thank P. Hennequin for fruitful discussions. This work has been carried out within the framework of the EUROfusion Consortium and has received funding from the Euratom research and training programme 2014-2018 under grant agreement No 633053. This work was also supported by the European Commission within the framework of the Erasmus Mundus International Doctoral College in Fusion Science and Engineering (FUSION-DC). The views and opinions expressed herein do not necessarily reflect those of the European Commission. This work was also performed within the framework of the Helmholtz Virtual Institute on Plasma Dynamical Processes and Turbulence Studies using Advanced Microwave Diagnostics. 


\section{References}

[1] A. J. Wootton et al., Phys. Fluids B 2, 2879 (1990)

[2] J. W. Connor, Plasma Phys. Control. Fusion 35, B293 (1993)

[3] P. H. Diamond et al., Plasma Phys. Control. Fusion 47, R35 (2005)

[4] T. S. Hahm et al., Phys. Plasmas 6, 922 (1999)

[5] N. Winsor et al., Phys. Fluids 11, 2448 (1968)

[6] G. R. McKee et al., Plasma Phys. Control. Fusion 48, S123 (2006)

[7] G. D. Conway et al., Plasma Phys. Control. Fusion 50, 085005 (2008)

[8] A. Fujisawa, Nucl. Fusion 49, 013001 (2009)

[9] P. M. Schoch et al., Bull. Am. Phys. Soc. 46, 8 (2001)

[10] A. Krämer-Flecken et al., Phys. Rev. Lett. 97, 045006 (2006)

[11] G. D. Conway et al., Plasma Phys. Control. Fusion 47, 1165 (2005)

[12] L. Vermare et al., Nucl. Fusion 52, 063008 (2012)

[13] C. A. de Meijere et al., Plasma Phys. Control. Fusion 56, 072001 (2014)

[14] G. D. Conway et al., Plasma Phys. Control. Fusion 50, 055009 (2008)

[15] A. Storelli et al., Phys. Plasmas 22, 062508 (2015)

[16] Z. Y. Qiu et al., Plasma Phys. Control. Fusion 51, 012001 (2009)

[17] Z. Gao, Plasma Science and Technology 13, 15 (2011)

[18] H. Sugama and T. H. Watanabe, J. Plasma Physics 72, 825 (2006)

[19] P. Angelino et al., Phys. Plasmas 15, 062306 (2008)

[20] M. Hirsch et al., Plasma Phys. Control. Fusion 43, 1641 (2001

[21] G. D. Conway et al., Plasma Fusion Res. 5, S2005 (2010)

[22] G. D. Conway et al., Plasma Phys. Control. Fusion 46, 951 (2004)

[23] C. Tröster, Ph. D. Thesis, Ludwig-Maximilian University, Munich (2008)

[24] E. Poli et al., Comput. Phys. Commun. 136, 90 (2001)

[25] J. Schirmer et al., Plasma Phys. Control. Fusion 49, 1019 (2007)

[26] M. Reich et al., Plasma Phys. Control. Fusion 46, 797 (2004)

[27] R. O. Schmidt, IEEE Trans. Antennas and Propagation AP-34, 276 (1986)

[28] J. W. Connor and L. Chen, Phys. Fluids 28, 2201 (1985)

[29] P. J. McCarthy, Phys. Plasmas 6, 3554 (1999)

[30] Z. Gao et al., Nucl. Fusion 49, 045014 (2009)

[31] Z. Gao, Phys. Plasmas 17, 092503 (2010)

[32] F. Zonca and L. Chen, EPL 83, 35001 (2008)

[33] Z. Qiu et al., Phys. Plasmas 22, 042512 (2015)

[34] M. Sasaki et al., Plasma Fusion Res. 8, 1403010 (2013)

[35] R. Singh et al., Plasma Phys. Control. Fusion 57, 125002 (2015)

[36] T. L. Rhodes et al., Phys. Plasmas 9, 2141 (2002)

[37] G. D. Conway, Plasma Phys. Control. Fusion 50, 124026 (2008)

[38] T. Watari et al., Phys. Plasmas 12, 062304 (2005)

[39] Z. Gao et al., Phys. Plasmas 15, 072511 (2008)

[40] Z. Gao, Phys. Plasmas 20, 032501 (2013)

[41] A. Biancalani et al., Nucl. Fusion 54, 104004 (2014)

[42] H. Sugama and T. H. Watanabe, J. Plasma Physics 74, 139 (2008)

[43] P. Simon et al., Proc. 11th International Reflectometry Workshop, Palaiseau (2013)

[44] J. C. Hillesheim et al., Phys. Plasmas 19, 022301 (2012)

[45] S. V. Novakovskii et al., Phys. Plasmas 4, 4272 (1997)

[46] J. R. Robinson et al., Plasma Phys. Control. Fusion 54, 105007 (2012)

[47] A. Yu. Yashin et al., Nucl. Fusion 54, 114015 (2014)

[48] H. Ren, Phys. Plasmas 21, 044505 (2014)

[49] V.P. Lakhin et al., Phys. Lett. A 374, 4872 (2010) 
[50] J. Yu and X. Gong, Nucl. Fusion 53, 123027 (2013) 